\title{
Identification of blood-based molecular signatures for prediction of response and relapse in schizophrenia patients
}

\author{
E Schwarz ${ }^{1}$, PC Guest ${ }^{1}$, J Steiner ${ }^{2}$, B Bogerts ${ }^{2}$ and S Bahn ${ }^{1,3}$
}

The current inability of psychiatric medicine to objectively select the most appropriate treatment or to predict imminent relapse are major factors contributing to the severity and clinical burden of schizophrenia. We have previously used multiplexed immunoassays to show that schizophrenia patients have a distinctive molecular signature in serum compared with healthy control subjects. In the present study, we used the same approach to measure biomarkers in a population of 77 schizophrenia patients who were followed up over 25 months with four aims: (1) to identify molecules associated with symptom severity in antipsychotic naive and unmedicated patients, (2) to determine biomarker signatures that could predict response over a 6-week treatment period, (3) to identify molecular panels that could predict the time to relapse in a cross-sectional population of patients in remission and (4) to investigate how the biological relapse signature changed throughout the treatment course. This led to identification of molecular signatures that could predict symptom improvement over the first 6 weeks of treatment as well as predict time to relapse in a subset of 18 patients who experienced recurrence of symptoms. This study provides the groundwork for the development of novel objective clinical tests that can help psychiatrists in the clinical management of schizophrenia. Translational Psychiatry (2012) 2, e82; doi:10.1038/tp.2012.3; published online 21 February 2012

\section{Introduction}

Less than $50 \%$ of schizophrenia patients respond to an initial treatment with antipsychotic medication. ${ }^{1,2}$ This is not surprising as most of the current antipsychotic medications used in the treatment of schizophrenia were discovered empirically based on serendipitous clinical observations and reverse pharmacology. ${ }^{3}$ Therefore, there is not sufficient understanding of the underlying pathophysiology of schizophrenia to inform diagnosis. Furthermore, traditional pharmacotherapy for schizophrenia using 'blockbuster' drugs usually leads to the administration and switching of drugs multiple times until an adequate response is achieved. It is perhaps not surprising that there is a low treatment response rate and a high rate of non-compliance often resulting in relapse. ${ }^{4}$ Thus, reliable tests with a sound biological rationale to guide the treatment selection are needed, as recently suggested in the case of antidepressant research and development. ${ }^{5}$

The adoption of new, molecule-based algorithms has not been common practice in neuropsychiatric research. However, this is likely to change considering the increased drive for the improved clinical management and benefit/cost ratios in schizophrenia treatment, as well as the Food and Drug Administration's integrated objective for personalized medicine approaches in managing diseases. ${ }^{6}$ Cost containment for clinical trials will also be a sufficient incentive for pharmaceutical companies to pursue this approach. ${ }^{7}$
We have previously used multiplexed immunoassays to show that schizophrenia patients have a distinctive molecular signature in blood serum. ${ }^{8,9}$ In this study, we have used the same method in an attempt to identify molecular serum signatures that could predict response and subsequent relapse in 77 schizophrenia patients. The study had four main aims: (1) to identify molecules associated with symptom severity in antipsychotic naive and unmedicated patients, (2) to determine biomarker signatures that could predict response over a 6week treatment period, (3) to identify molecular panels that could predict the time to relapse in a cross-sectional population of patients in remission and (4) to investigate how the biological relapse signature changed throughout the treatment course. For this purpose, 77 initially acutely psychotic schizophrenia patients were treated for 6 weeks and followed up until relapse occurred within a 2-year time period.

\section{Methods}

Clinical samples. The institutional ethical committee of the University of Magdeburg approved the protocols of the study, informed written consent given by all participants and studies were conducted according to the Declaration of Helsinki. All diagnoses were carried out using the Diagnostic and Statistical Manuel (DSM)-IV, and clinical tests were performed by psychiatrists under good clinical practice-compliance to minimize variability. Subjects were diagnosed as having the

\footnotetext{
${ }^{1}$ Department of Chemical Engineering and Biotechnology, University of Cambridge, Cambridge, UK; ${ }^{2}$ Department of Psychiatry, University of Magdeburg, Magdeburg, Germany and ${ }^{3}$ Department of Neuroscience, Erasmus Medical Centre, Rotterdam, The Netherlands

Correspondence: Dr E Schwarz, Department of Chemical Engineering and Biotechnology, University of Cambridge, Institute of Biotechnology, Tennis Court Road, Cambridge CB2 1QT, Cambridge, UK or S Bahn, Department of Neuroscience, Erasmus Medical Centre, NL-3000 CA, Rotterdam, The Netherlands.

E-mail: es404@cam.ac.uk or sb209@cam.ac.uk

Keywords: biomarkers; proteomics; relapse; response; schizophrenia

Received 16 August 2011; revised 15 November 2011; accepted 8 January 2012
} 
paranoid subtype of schizophrenia (295.30). The exclusive focus on paranoid schizophrenia, which comprises the most prevalent subtype of the illness, was intended to minimize variability. Out of 77 patients, 36 were in the first stage of illness and antipsychotic naïve at the start of the study (Table 1). The remaining 41 patients had been antipsychotic-free for at least 6 weeks. At T0, the average disease duration was $6.32 \pm 8.63$ years. All subjects were treated as inpatients over the first 6 weeks of treatment. Clinicians had access to all detailed clinical files including medical histories by proxy and referral letters from the general practitioners. Any patients with other medical conditions such as type 2 diabetes, hypertension, cardiovascular or autoimmune diseases were excluded. Patients with autoimmune diseases were excluded to rule out psychosis secondary to disorders like systemic lupus erythematodes or multiple sclerosis, which may cause schizophrenia-like psychotic symptoms but in the view of the authors rather resemble organic schizophreniform psychosis than schizophrenia.

During the first 6 weeks of treatment, where all subjects were treated as inpatients, therapeutic drug monitoring from peripheral blood samples was applied in order to assess the compliance.

Blood samples were collected from all subjects by venous puncture into S-Monovette $7.5 \mathrm{ml}$ serum tubes (Sarstedt; Numbrecht, Germany) at the start of the study (TO), after the 6 week treatment period (T6), at the last clinical visit before relapse (TR) and during relapse (Figure 1). Serum was prepared by placing samples at room temperature for $2 \mathrm{~h}$ to allow blood coagulation, followed by centrifugation at $4000 \mathrm{~g}$ for $5 \mathrm{~min}$ to remove particulate material. The resulting supernatants were stored at $-80^{\circ} \mathrm{C}$ in Low Binding Eppendorf tubes (Hamburg, Germany) before the analysis.

Multiplex immunoassay. The HumanMAP immunoassay platform was used to measure the serum concentrations of 191 proteins and small molecules (Supplementary Table 1) in a Clinical Laboratory Improvement Amendments-certified laboratory at Rules Based Medicine (Austin, TX, USA) as described previously. ${ }^{9}$ Samples were randomized and blinded to analysts using code numbers until all biochemical assays were complete. Assays were calibrated using standards, raw intensity measurements converted to absolute protein concentrations by comparison to the standards, and performance was verified using quality control samples. Data analyses were performed using the statistical software package R (http://www.r-project.org). The protocol for the study participants, clinical samples and test methods was carried out in compliance with the Standards for Reporting of Diagnostic Accuracy (STARD) initiative. ${ }^{10}$

Data analysis. The molecular data set was preprocessed by filtering out all analytes, which contained measurements outside the limits of quantitation in more than $30 \%$ of samples. After this procedure, 168 out of 191 analytes remained in the data set, and the proportion of missing values dropped from 16.7 to $6.8 \%$. To assess data quality, Principal Component Analysis (SIMCA-P+ vs 12.0, Umetrics, Umeå, Sweden) was used for the detection of strong effects on the overall variance in the filtered data set. No such effects could be identified across the first 10 principal components, which covered $44 \%$ of the variance in the data set. Shapiro-Wilk analysis showed that the majority of the investigated analytes (97\%) were significantly $(P<0.05)$ non-normally distributed. Outliers in each analyte as well as response variables were excluded if a given concentration differed by more than two standard deviations from the mean of the respective analyte. For the different analyses described in the present study, this resulted in the removal of $3.2-4.1 \%$ of subjects per analyte, on average. Outliers identified in this fashion did not substantially affect results, as repetition of all analyses without exclusion of outliers led to qualitatively identical results.

Significant associations were determined using non-parametric Spearman's correlation tests and adjusted for false

Table 1 Demographic details of schizophrenia patients at T0 and at the time of relapse

\begin{tabular}{|c|c|c|c|}
\hline & At TO & At T6 & At relapse \\
\hline $\begin{array}{l}n \\
\text { Sex (m/f) } \\
\text { Age (years) } \\
\left.\text { BMl (kg m }{ }^{-2}\right) \\
\text { Waist circumference }(\mathrm{cm}) \\
\text { Hip circumference }(\mathrm{cm}) \\
\text { Blood pressure, diastolic }(\mathrm{mm} \mathrm{Hg}) \\
\text { Blood pressure, systolic }(\mathrm{mm} \mathrm{Hg}) \\
\text { Pulse (heart beats per min) }\end{array}$ & $\begin{array}{c}77 \\
50 / 27 \\
35.56 \pm 11.06 \\
25.35 \pm 4.61 \\
91.08 \pm 14.42 \\
101.20 \pm 10.04 \\
80.65 \pm 11.46 \\
129.18 \pm 14.91 \\
81.81 \pm 12.44\end{array}$ & $\begin{array}{c}77 \\
50 / 27 \\
- \\
26.12 \pm 4.50 \\
93.55 \pm 13.31 \\
103.22 \pm 9.29 \\
76.68 \pm 10.80 \\
121.36 \pm 14.68 \\
77.84 \pm 12.41\end{array}$ & $\begin{array}{c}18 \\
12 / 6 \\
40.44 \pm 11.89 \\
26.23 \pm 5.28 \\
92.56 \pm 11.92 \\
102.72 \pm 8.78 \\
80.28 \pm 9.31 \\
128.89 \pm 14.61 \\
87.78 \pm 15.73\end{array}$ \\
\hline $\begin{array}{l}\text { PANSS P1-P7 (score) } \\
\text { PANSS N1-N7 (score) } \\
\text { PANSS G1-G16 (score) } \\
\text { HAMD (score) }\end{array}$ & $\begin{array}{l}22.97 \pm 7.17 \\
19.33 \pm 8.69 \\
42.99 \pm 10.33 \\
17.43 \pm 9.59\end{array}$ & $\begin{array}{r}12.68 \pm 5.30 \\
14.36 \pm 6.83 \\
28.69 \pm 9.09 \\
7.08 \pm 6.94\end{array}$ & $\begin{array}{l}22.11 \pm 6.11 \\
17.67 \pm 8.24 \\
38.83 \pm 13.44 \\
19.33 \pm 11.66\end{array}$ \\
\hline $\begin{array}{l}\text { Smoking (cigarettes per day) } \\
\text { Cannabis (yes/no) }\end{array}$ & $\begin{array}{l}9.47 \pm 9.10 \\
16 / 61\end{array}$ & $\begin{array}{l}9.57 \pm 9.49 \\
3 / 74\end{array}$ & $\begin{array}{l}10.56 \pm 10.09 \\
5 / 13\end{array}$ \\
\hline $\begin{array}{l}\text { Medication (olanzapine/quetiapine/risperidone/other) } \\
\text { Cumulative chlorpromazine units (mg) }\end{array}$ & $\overline{0}$ & $\begin{array}{c}18 / 14 / 24 / 21 \\
17123 \pm 12657\end{array}$ & \\
\hline
\end{tabular}

Abbreviations: HAMD, Hamilton D; PANSS, Positive and Negative Syndrome Scale.

Values are shown as mean \pm s.d. 


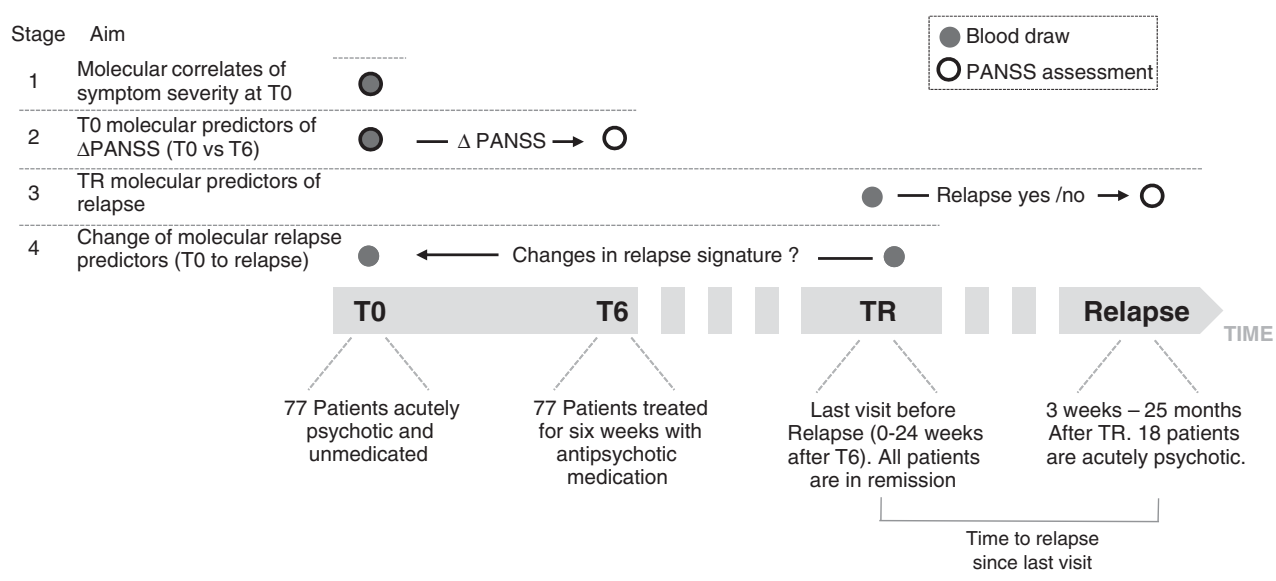

Figure 1 Schematic diagram showing time of sampling of schizophrenia patients treated with antipsychotics. Subjects $(n=77)$ were treated for 6 weeks (T0-T6) with antipsychotics. After this, 0-24 weeks elapsed until the last clinical visit (TR) of the 18 patients who relapsed after an additional 3 weeks to 25 months. Blood samples were drawn from subjects, serum prepared at the indicated time points and the levels of 190 proteins and small molecules were measured by multiplex immunoassay.

discovery rate. ${ }^{11}$ Comparisons between the two groups were based on the non-parametric Wilcoxon Rank sum test or the paired Wilcoxon Rank sum test for within-subject comparisons. Comparisons between three or more groups were performed using the non-parametric Kruskal-Wallis test. Analysis of co-variance (ANCOVA) was used to account for 'regression to the mean' effects between baseline and follow-up measurements. ${ }^{12}$ ANCOVA was also used to estimate the effect of body mass index (BMI) on molecular differences between the groups. For ANCOVA, all variables were $\log _{10}$-transformed to approximate normality. Unadjusted $P$ values of less than 0.05 were considered as significant.

To identify a molecular fingerprint that could predict patient responses such as time to relapse, we applied Random Forests analysis. ${ }^{13}$ This technique works by building multiple decision trees and using a majority decision of classification outputs among trees to assign patients to one of two groups based on the levels of serum molecules. This method was also used to select the most important molecules for the prediction. For this purpose, the measured values of each molecule were permuted sequentially and the variable importance was determined from the impact of such randomization on the classification output.

\section{Results and Discussion}

Experimental design. As already mentioned in the introduction, Multiplex immunoassay analysis was carried out in four stages (Figure 1):

- The first stage was aimed at the identification of molecules that correlated with symptom severity in untreated schizophrenia patients. For this purpose, serum was obtained from 77 patients who were experiencing an episode of acute psychosis (T0). At this time-point, all patients were unmedicated or antipsychotic naive. All 77 patients were followed up over a subsequent 6-week treatment period with antipsychotic medication.
- The change in symptom severity between T0 and the end of the 6 week follow-up period (T6) was the main outcome variable for the second stage of this study. Here, we tried to identify molecules in the TO samples that predicted the change in the symptom severity between T0 and T6.

- For the third stage of the study, we measured serum molecules at TR, the last clinical visit before patients relapsed. At this time-point patients were in remission with different durations of antipsychotic treatment. TR was chosen since patients at this time-point reflected the real clinical scenario of a heterogeneous patient population for which molecular relapse predictors would be most useful. The aim of this stage was to identify a molecular signature that could predict the time to relapse for subjects in this cross-sectional group.

- During the fourth stage, we investigated how the molecular relapse signature changed throughout the treatment period.

Patient characteristics and psychopathology scores. Patient characteristics are indicated in Table 1. As the symptom severity was the main outcome variable for stages 1-3 of this study, we tested whether this was associated with any of the remaining recorded patient characteristics. This was done to identify covariates that could influence the identification of potential biomarkers. At T0, no significant associations between PANSS scores and the remaining patient characteristics were found (Table 2). Secondly, there were no significant associations between patient characteristics that were not related to symptom severity and the change in PANSS between T0 and T6 (DPANSS) (Table 2).

$\triangle$ PANSS quantifies the symptom improvement that patients experienced after 6 weeks of antipsychotic treatment. On average, positive PANSS scores decreased from 23.0 to $12.7(P<0.001$; paired Wilcoxon test) and negative PANSS scores from 19.3 to $14.4(P<0.001)$ (Figure 2). Patients with more severe symptoms at T0 improved more than those with less severe symptoms. This is likely to have been influenced 
by 'regression to the mean', as patients with severe symptoms are statistically more likely to have a reduction in these symptoms at the follow-up time-point. ${ }^{14}$ Therefore, $\Delta$ positive PANSS was significantly correlated to the TO PANSS values ( $P<0.001, \rho=0.77$; Spearman correlation) (Figure 2). This was also the case for negative PANSS scores $(P<0.001$, $\rho=0.65)$. As a consequence of the difference in choice of antipsychotic medication for patients with differing symptom

Table 2 Association between patient characteristics at TO and symptom severity

\begin{tabular}{lccccc}
\hline & \multicolumn{2}{c}{ TO PANSS } & & \multicolumn{2}{c}{ APANSS } \\
\cline { 2 - 3 } \cline { 5 - 6 } Data at T0 & Positive & Negative & & Positive & Negative \\
\hline Age & $0.0747^{\mathrm{a}}$ & $0.6270^{\mathrm{a}}$ & & $0.3552^{\mathrm{a}}$ & $0.4529^{\mathrm{a}}$ \\
Gender & $0.2905^{\mathrm{b}}$ & $0.7382^{\mathrm{b}}$ & & $0.7340^{\mathrm{b}}$ & $0.3971^{\mathrm{b}}$ \\
BMI & $0.1990^{\mathrm{a}}$ & $0.4599^{\mathrm{a}}$ & & $0.3092^{\mathrm{a}}$ & $0.5675^{\mathrm{a}}$ \\
Waist-circumference & $0.2935^{\mathrm{a}}$ & $0.4939^{\mathrm{a}}$ & & $0.4857^{\mathrm{a}}$ & $0.8832^{\mathrm{a}}$ \\
Smoking & $0.9021^{\mathrm{a}}$ & $0.3390^{\mathrm{a}}$ & & $0.6040^{\mathrm{a}}$ & $0.6175^{\mathrm{a}}$ \\
Treatment state at T0 & $0.2785^{\mathrm{b}}$ & $0.2809^{\mathrm{b}}$ & & $0.8660^{\mathrm{b}}$ & $0.3960^{\mathrm{b}}$ \\
& & & & & \\
\hline
\end{tabular}

The second and third columns show significance ( $P$-values) for the correlations between patient characteristics and psychopathology (positive and negative PANSS items) at TO. The fourth and fifth columns show significance ( $P$-values) for the correlations between patient characteristics at TO and the change in PANSS ratings ( $\triangle$ PANSS) between T0 and T6.

aSpearman's correlation test.

bWilcoxon Rank Sum test. severity, patients treated with quetiapine improved significantly less than those treated with olanzapine or risperidone (positive PANSS: $P=0.001$, negative PANSS: $P=0.101$, Kruskal-Wallis test). Due to the higher risk of weight gain associated with olanzapine treatment, ${ }^{15}$ patients who were antipsychotic naive at T0 experienced a higher but not significant increase in BMI until T6 compared to patients treated with other medications $\left(\triangle \mathrm{BM} \mathrm{OLA}_{\mathrm{OA}}=1.1\right.$ in olanzapine group vs $\Delta \mathrm{BMI}_{\mathrm{OTHER}}=0.63$ in remaining patients, $P=0.200$, Wilcoxon test). This effect was absent in patients who were not antipsychotic-naive at $\mathrm{TO}\left(\Delta \mathrm{BMI}_{\mathrm{OLA}}=0.7, \Delta \mathrm{BMI}_{\mathrm{OTHER}}\right.$ $=0.8, P=0.845$ ), potentially due to the fact that patients who had already been treated with olanzapine had also a higher BMI compared with the remaining patients in this group ( $P=0.030$, Wilcoxon test). Across all 77 patients, there was also no significant difference between antipsychotic medications in the effect on $\Delta \mathrm{BMI}$ during the first 6 weeks of treatment, when patients were treated as inpatients ( $P=0.563$, Kruskal-Wallis test).

Overall, there was a greater decrease in positive symptoms (64\%) compared with that seen for negative symptoms (40\%), and this was reflected in the number of patients who showed these responses. For positive symptoms, 52 patients improved by more than $50 \%$. For negative symptoms, only 33 out of the 77 patients showed this improvement. The finding that T0 positive and negative PANSS scores were not
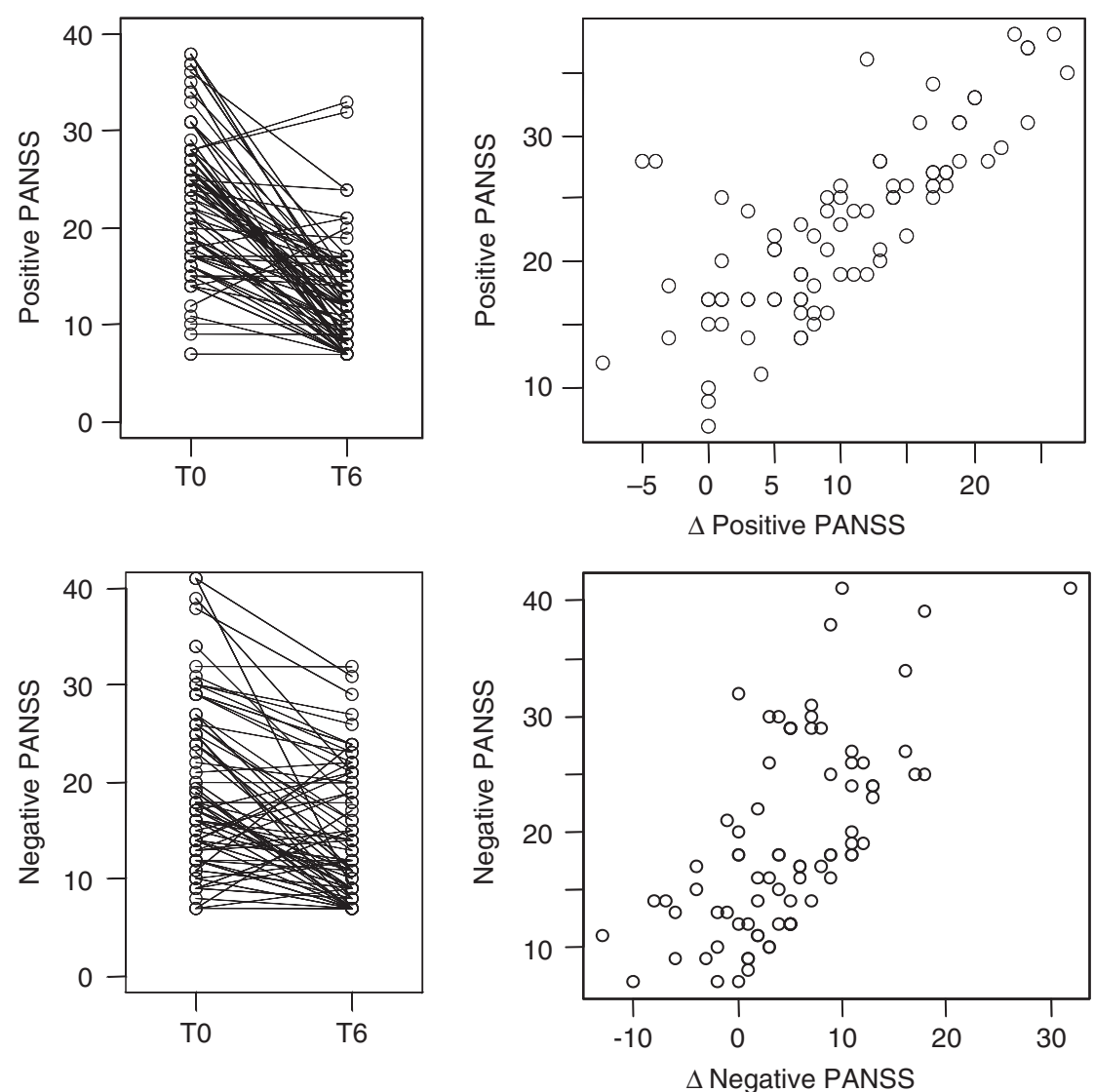

Figure 2 Symptom severity measured using PANSS scores during the first 6 weeks of treatment (T0-T6). PANSS positive (top left panel) and negative (bottom left panel) item scores improved significantly over the treatment period. The lines between T0 and T6 connect scores of the same patients. Right panels: the change in positive (top) and negative (bottom) PANSS scores were correlated to the corresponding symptom severity at TO. 
significantly correlated to each other ( $P=0.286$; Spearman correlation) suggested that these parameters were independent. Likewise, $\Delta$ positive and $\Delta$ negative PANSS scores were not significantly correlated $(P=0.094, \rho=0.19$; Spearman test).

Stage 1: identification of biomarkers of symptom severity at T0. During the first stage of the analysis, we attempted to identify any associations between TO PANSS scores and T0 levels of serum molecules. A total of 18 molecules were significantly correlated with positive T0 PANSS scores (14 with $\rho>0$ ) and 8 molecules (4 with $\rho>0$ ) were associated with negative T0 PANSS scores $(P<0.05$; Spearman correlation; Table 3 shows those associations with $P<0.01)$. The significant changes included increased levels of the acute phase response pathway proteins myoglobin, ferritin, C-reactive protein and interleukin 16, which have already been implicated in the pathophysiology of schizophrenia. ${ }^{16}$ In addition, prolactin levels showed a significant negative correlation $(\rho=-0.27$, $P=0.022$ ) with positive PANSS, indicating that patients with higher prolactin levels had lower symptom severity at TO. This is interesting as prolactin has been used as a biomarker of antipsychotic drug administration for many years, ${ }^{17}$ and we have shown recently that levels of this protein are elevated in serum from first onset antipsychotic naïve schizophrenia patients. ${ }^{18}$

Stage 2: identification of biomarkers for response prediction (DPANSS). In the second stage of this study, we attempted to identify molecules that could predict the improvement in PANSS scores between T0 and the end of the 6 weeks treatment period (T6). For this purpose, we determined associations between T0 molecular levels and $\triangle$ PANSS. Due to the 'regression to the mean' affecting $\triangle$ PANSS, we performed ANCOVA analysis using TO PANSS levels as covariate. ${ }^{12}$ This showed that no molecule was significantly correlated with $\Delta$ positive PANSS and only insulin showed a weak association with $\Delta$ negative PANSS $(P=0.006, Q=0.47$, Table 3$)$. Lower insulin levels at T0 were predictive of symptom improvement after antipsychotic treatment. This is consistent with previous studies, which have shown that antipsychotic-induced effects such as insulin resistance and weight gain are associated with and may even be required for symptom improvement in response to antipsychotic treatment. ${ }^{19,20}$

\section{Stage 3: identification of biomarkers for prediction of relapse}

Univariate predictors of relapse. The next stage of the analysis was aimed at the identification of a molecular signature that could predict the time until patients in remission would relapse. For this purpose, we analyzed the sera of 18 patients who relapsed after the treatment course (Table 1). Six patients were off medication at TR and all but three patients were off medication at the time of relapse. The time to relapse was quantified as the time between the last clinical visit (TR) and relapse. These values were dichotomized such that patients below the median (8.3 months) were partitioned into a short-term relapse group $(n=9)$ and those above the median were assigned to the long-term relapse group $(n=9)$. The mean times to relapse in the short- and long-term relapse groups were 3.7 and 13.2 months, respectively. The classification into short-term and long-term relapse groups was similar between the scenarios when time to relapse was determined from either T0 or TR (16 out of 18 subjects stayed in the same class). This showed that the assignment of patients to relapse groups was reasonably consistent throughout the treatment.

To avoid the effect of potential confounding factors on biomarkers during this stage, we tested the association between the two relapse groups and all recorded patient characteristics at TR. Subjects in the short-term relapse group had significantly lower BMls compared with those in the longterm relapse group $\left(24 \pm 4\right.$ and $28 \pm 4 \mathrm{~kg} \mathrm{~m}^{-2}$, respectively; $P=0.040$; Wilcoxon test) (Figure 3). This could have been

Table 3 Significant associations between molecular levels at T0 and psychopathology scores

\begin{tabular}{|c|c|c|c|c|c|c|c|c|}
\hline \multirow[b]{3}{*}{ Molecule } & \multicolumn{4}{|c|}{ TO PANSS } & \multicolumn{4}{|c|}{$\triangle P A N S S$ (ANCOVA) } \\
\hline & \multicolumn{2}{|c|}{ Positive } & \multicolumn{2}{|c|}{ Negative } & \multicolumn{2}{|c|}{ Positive } & \multicolumn{2}{|c|}{ Negative } \\
\hline & P-value & $\rho$ & P-value & $\rho$ & P-value & $\rho$ & P-value & $\rho$ \\
\hline Fatty acid-binding protein & $<0.001$ & 0.45 & & & & & & \\
\hline FGF-basic & $<0.001$ & -0.41 & 0.003 & -0.34 & & & & \\
\hline Interleukin 16 & $<0.001$ & 0.40 & & & & & & \\
\hline Creatine kinase MB & 0.001 & 0.40 & & & & & & \\
\hline Myoglobin & 0.002 & 0.36 & & & & & & \\
\hline C-reactive protein & 0.003 & 0.34 & & & & & & \\
\hline NGAL & 0.004 & 0.35 & & & & & & \\
\hline Matrix metalloproteinase 2 & 0.005 & -0.33 & 0.010 & -0.30 & & & & \\
\hline Ferritin & 0.008 & 0.32 & & & & & & \\
\hline Hepatocyte growth factor & 0.009 & 0.31 & & & & & & \\
\hline Osteopontin & & & 0.008 & 0.32 & & & & \\
\hline Amphiregulin & & & 0.009 & 0.30 & & & & \\
\hline Insulin & & & & & & & 0.005 & -0.37 \\
\hline
\end{tabular}

Abbreviations: FGF, fibroblast growth factor-basic; NGAL, neutrophil gelatinase-associated lipocalin

Columns 2 to 5 show significance ( $P$-value) and correlation coefficients $(\rho)$ for the association between molecular levels and symptom severity for positive and negative PANSS scores at T0. Columns 4 to 7 show the corresponding estimates for T0 molecular levels with $\triangle$ PANSS (using T0 PANSS levels as covariate). $P$-values $<0.01$ are shown 

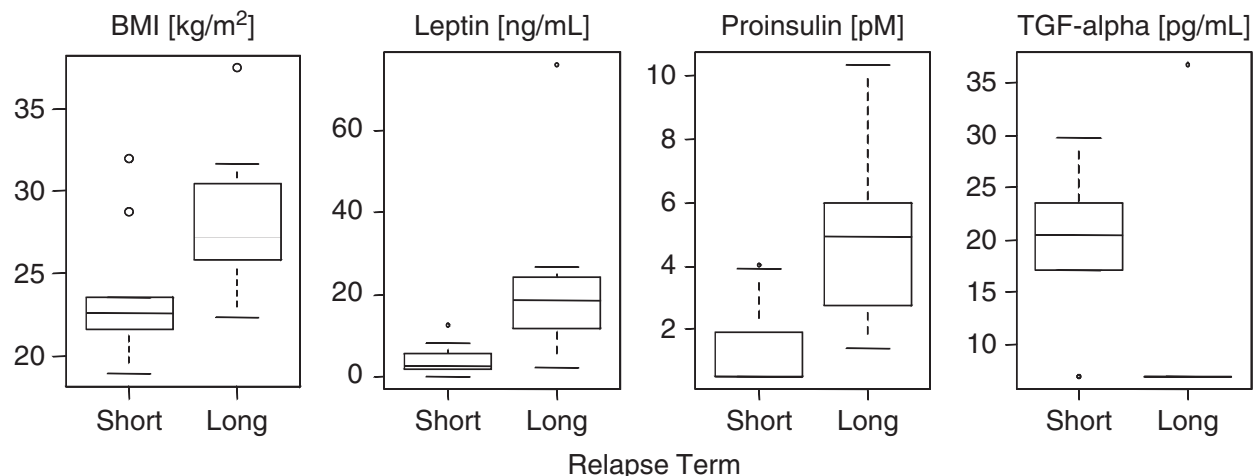

Figure 3 Differences of BMI and the levels of leptin, proinsulin and TGF- $\alpha$ between the short- and long-term relapse groups. The measurements were taken at the last clinical visit before relapse (TR).

caused by non-compliance of patients in the short-term relapse group. The difference in BMls did not reach significance at the actual time of relapse $(P=0.0625)$. The two groups did not differ in age ( $P=0.232$, Wilcoxon test), gender $(P=0.577$, Fisher's exact test), PANSS positive ( $P=0.894$, Wilcoxon test) or PANNS negative $(P=0.824)$ scores. We found similar distributions of medication prescribed at TO (the number of subjects receiving olanzapine/ olanzapine + risperidone/risperidone/quetiapine/haloperidol/ none was $2 / 2 / 2 / 2 / 0 / 1$ in the short-term relapse group and $5 / 0$ / $1 / 1 / 1 / 1$ in the long-term group). There was no significant difference in time to relapse between olanzapine-treated patients and those receiving other medications $(P=0.348$ in short-term and $P=0.111$ in long-term relapse group, $P=0.717$ in all 18 patients). Also, we did not find an association of time to relapse with medication status at TR ( $P=0.708$, Wilcoxon test). This indicated that the six patients who were off medication at TR were equally distributed across the short- and long-term relapse groups. Furthermore, there was no difference in time to relapse between patients who were antipsychotic naive or unmedicated at T0 $(P=0.328$, Wilcoxon test). This showed that no clinical feature except for $\mathrm{BMI}$ was indicative of the time to relapse at the last clinical visit. Although time to relapse was not associated with medication status at the last clinical visit, there is a possibility that the difference in relapse time was associated with noncompliance rather than the molecular factors reflecting response. This is addressed specifically in stage 4.

To identify molecules associated with the time of relapse, we compared the levels of all molecules at TR with respect to subjects who ended up in the short- or long-term relapse groups using non-parametric Wilcoxon tests. This analysis showed that 27 molecules were significantly different between the groups $(P<0.05, Q<0.31)$. To determine if the significant difference in BMI values at TR had any effect on the levels of these molecules, we performed ANCOVA. A total of 13 of these molecules remained significant $(P<0.05)$ after this analysis and 11 of the molecules were also significant using non-parametric Wilcoxon tests (Table 4). The three molecules with the greatest differences between the short- and long-term relapse groups were leptin, proinsulin and transforming growth factor (TGF)-alpha (Figure 3).
Table 4 Significant molecular differences at the last clinical visit before relapse (TR) between the short- and long-term relapse groups

\begin{tabular}{lccc}
\hline Analyte & P-value & q-value & $\boldsymbol{R C}$ \\
\hline Leptin $^{\mathbf{a}}$ & 0.033 & 0.559 & 0.18 \\
Proinsulin $^{\mathbf{a}}$ & 0.021 & 0.559 & 0.29 \\
Transforming growth factor alpha $^{\mathbf{a}}$ & 0.018 & 0.559 & 1.85 \\
$\boldsymbol{\beta}_{\text {-Cellulin }}^{\mathbf{a}}$ & 0.015 & 0.559 & 0.57 \\
CD5 L & 0.002 & 0.335 & 0.63 \\
Matrix metalloproteinase 9 $_{\text {CD40 }^{\mathbf{a}}}$ & 0.029 & 0.559 & 1.40 \\
Macrophage-derived chemokine $_{\text {Brain-derived neurotrophic factor }}$ & 0.011 & 0.559 & 0.74 \\
Apolipoprotein Cl $^{\mathbf{a}}$ & 0.021 & 0.559 & 0.74 \\
Matrix metalloproteinase 7 & 0.042 & 0.559 & 0.77 \\
$\boldsymbol{\beta}_{\text {-2-Microglobulin }}$ & 0.046 & 0.559 & 0.78 \\
Tumor necrosis factor receptor like 2 & 0.045 & 0.559 & 0.79 \\
& 0.046 & 0.559 & 0.80 \\
& 0.039 & 0.559 & 0.85
\end{tabular}

All molecular differences had a $P$-value of less than 0.05 after ANCOVA using $\mathrm{BMI}$ at TR as covariate. The $q$-values represent $P$-values adjusted for False Discovery Rate. The RC is the ratio change between the short-term and long term-relapse groups at TR. Molecules shown in bold were also significant using non-parametric Wilcoxon tests.

${ }^{\mathrm{a}}$ Molecules selected as important predictors in a multivariate Random Forest model.

The significant association between time to relapse and $\mathrm{BMI}$, and the finding of higher BMls in the long-term relapse group, provided further evidence for the importance of metabolic changes such as weight gain in antipsychotic treatment response. ${ }^{21,22}$ Also, leptin and proinsulin have been used previously as biomarkers for insulin resistance ${ }^{23}$ and metabolic syndrome. ${ }^{24}$ These findings raise the interesting possibility that schizophrenia patients who experience little or no increase in body weight or in the levels of these molecules in response to antipsychotic treatment are more likely to relapse. Previous studies have indicated that the levels of leptin $^{25}$ and insulin ${ }^{26}$ are positively correlated with BMI in patients treated with antipsychotics. However, it is not clear whether the changes in these two hormones are involved in the mechanism of action of antipsychotics, if they are due to side effects or if they are a consequence of changes that occur in the brain. Previous studies have found that high levels of insulin can lead to impaired cognition and administration of insulin-sensitizing agents can reverse this effect. ${ }^{27}$ Also, it has been shown that leptin levels are inversely correlated with 
grey matter volumes in obese subjects, suggesting that high levels of this hormone can also have negative effects on the brain function. ${ }^{28}$

The final objective of stage 3 was an attempt to predict the time to relapse using serum molecular signatures from the last clinical visit (TR). The Random Forests technique was used to select molecules that were the most important determinants for the prediction of relapse time (short- or long-term) and for determining the accuracy of this prediction. This led to the selection of a group of molecules comprised of leptin, proinsulin, TGF- $\alpha$, $\beta$-cellulin, CD5L, CD40 and Apo Cl, which were also significant in the univariate analysis (see above), as well as clusterin, insulin, interleukin-8, MIP-1- $\beta$ and matrix metalloproteinase 3 . Prediction testing using this combination of molecules yielded an accuracy of $94.5 \%$ in determining whether subjects were in the short- or long-term relapse groups.

We also tested the BMI values at the last clinical visit (TR) as a potential predictor of relapse time. Interestingly, another round of Random Forest analysis did not result in selection of BMI as an important variable, although 11 out of the 12 molecules indicated above were reselected (excluding matrix metalloproteinase 3). BMI alone achieved a predictive performance of $83.4 \%$, which was lower than that achieved using the signature of 12 molecules indicated above.

Stage 4: changes in the relapse biomarker signature induced by treatment. In the final stage of this study, we performed an exploratory analysis into the change in molecular predictors of relapse throughout the treatment period. For 15 of the 18 patients who experienced relapse, samples were available at T0 and T6. Similar to the findings at TR, patients in the short-term relapse group $(n=7)$ had a lower BMI at T0 compared to those in the long-term relapse group $(n=8)$, although this difference was not significant $(P=0.094$; Wilcoxon test). At T0, 8 molecules showed significantly different levels between the short- and longterm relapse groups $(P<0.05$, Wilcoxon test), including leptin $(P=0.040)$, although (Supplementary Table 1) all of these molecules had a high false discovery rate. This demonstrated that at T0 there were no pronounced molecular differences between the two relapse groups.

Therefore, we tested the multivariate biomarker signature identified above to determine if this was altered at T0. A Random Forests algorithm comprising these molecules was tested using T0 samples from the same patients. All patients who were detected in the short-term relapse group at TR were also identified at T0. Interestingly, the molecular signature at T0 also predicted imminent relapse for $50 \%$ of the patients (4 out of 8 ) in the long-term relapse group. Although based on a low n-number, this may suggest that the molecular state at T0 was more predictive of relapse than when measured at TR. This could indicate that at T0, when patients were unmedicated and acutely psychotic, their molecular profile was suggestive of imminent relapse. During the treatment the molecular profile might have responded in those patients, who would only relapse after a longer period of time.

With this in mind, we analyzed whether serum molecules of subjects in short- and long-term treatment groups showed differences in early response to medication. For this purpose, the change in serum levels of molecules between T0 and T6 was compared between the two relapse groups. ANCOVA analysis was performed using baseline molecular levels as covariates to prevent a potential 'regression to the mean' on results. ${ }^{12}$ We found that eight molecules were associated with the time of relapse $(P<0.05)$. For example, the early treatment response of leptin, insulin and C-peptide were significantly different between the relapse groups $(P<0.041$; $Q=0.52$, Figure 4). Levels of these three molecules showed essentially no change in the group that relapsed early, although these were increased significantly in patients who relapsed later. Other molecules that showed a differential early treatment response between the relapse groups are listed in Supplementary Table 2. In addition, the short-term relapse group had a lower $B M I$ increase $\left(\triangle B M I=0.28 \mathrm{~kg} \mathrm{~m}^{-2}\right)$ between $\mathrm{T} 0$ and $\mathrm{T} 6$ compared with the patients who relapsed later $\left(\Delta \mathrm{BMI}=1.94 \mathrm{~kg} \mathrm{~m}^{-2}\right)$. These findings support the hypothesis stated above that early response to medication, which affects metabolic and other processes, are related to predisposition for subsequent relapse. The result is of particular importance for the present study, as patients were
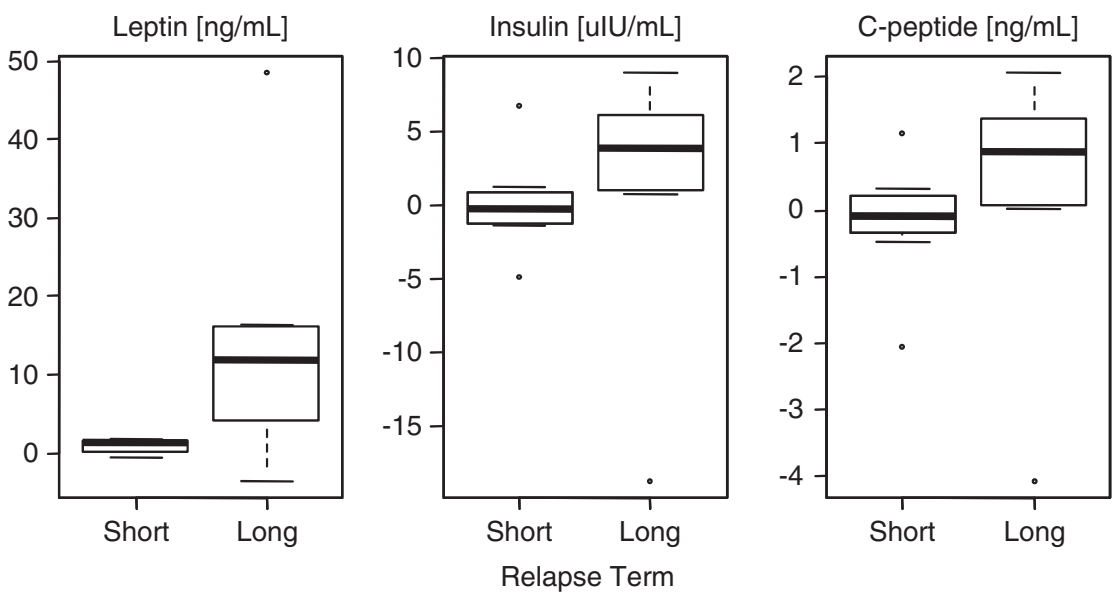

Figure 4 Differential early response to treatment (between T0 and T6) of leptin, insulin and C-peptide between the short- and long-term relapse groups. The figure shows the difference in the change of levels between T0 and T6 in serum from patients in the two different relapse groups. 
Table 5 Significant molecular differences at the time of relapse between the short- and long-term relapse groups

\begin{tabular}{lccc}
\hline Analyte & P-value & q-value & $\boldsymbol{R C}$ \\
\hline Leptin & 0.024 & 0.551 & 0.21 \\
Proinsulin total & 0.014 & 0.551 & 0.31 \\
Proinsulin & 0.034 & 0.551 & 0.39 \\
Creactive protein & 0.031 & 0.551 & 0.43 \\
Insulin & 0.024 & 0.551 & 0.50 \\
Interleukin 12 subunit p40 & 0.014 & 0.551 & 0.58 \\
Pulmonary activation-regulated chemokine & 0.024 & 0.551 & 0.58 \\
C peptide & 0.027 & 0.551 & 0.59 \\
Macrophage inflammatory protein 1- $\beta$ & 0.024 & 0.551 & 0.65 \\
CD40 & 0.014 & 0.551 & 0.78 \\
Thrombospondin 1 & 0.030 & 0.551 & 1.36 \\
Osteopontin & 0.038 & 0.565 & 1.39
\end{tabular}

All analytes are shown that had a $P$-value of less than 0.05 . The $q$-values represent $P$-values adjusted for the False Discovery Rate. The RC is the ratio change between the short-term and long term-relapse groups at the time of relapse.

inpatients between T0 and T6 and non-compliance could be ruled out by therapeutic drug monitoring. The differential early molecular response to medication may open up the possibility for the development of molecular tests that allow assessment of states indicative of relapse and identify those subjects who may require a change in treatment strategy.

As a final step of stage 4 , we investigated the molecular state in samples taken from subjects at the time of relapse. This revealed that 12 serum molecules had significantly different concentrations between the two relapse groups (Table 5). Again, abnormalities were pronounced for leptin, insulin, proinsulin and C-peptide, consistent with differences in BMI between the relapse groups and the suggestion of altered insulin signalling as seen by an increased insulin/ glucose ratio.

\section{Conclusion}

Non-compliance is a serious issue in the treatment of psychiatric conditions, such as schizophrenia given that this is the most common cause leading to psychotic relapse. This is the first study to identify serum molecules that may be indicative of relapse. Further development of assays targeting these specific molecules could lead to the development of useful tests to guide the clinical management of schizophrenia. As these molecular signatures may be dynamic and change over the course of treatment, we also investigated the change in molecular levels at the initiation of treatment up to and including the time of relapse. The results suggested that molecular processes associated with weight gain and metabolism changes have a central role in re-occurrence of psychotic symptoms. Specifically, a lower BMI and nonresponse of the molecular signature to treatment was associated with a shorter time to relapse. The identification of biomarkers, such as the insulin-related molecules proinsulin, C-peptide and insulin, along with changes in the hormone leptin, is consistent with previous studies showing changes in these molecules in first onset antipsychotic naive schizophrenia patients. ${ }^{18,29,30}$ We have also identified molecular signatures associated with symptom severity and response. However, the prediction of positive symptom improvement was of limited value due to the high proportion of patients in this study who showed an improvement in response to treatment.

Our results extend our previous findings that schizophrenia is characterized by a reproducible, peripheral signature. Further testing of these findings in validation studies using larger numbers of subjects in prospective clinical trials are required for the development of molecular tools, which could be useful in guiding the clinical management of schizophrenia. This could be particularly important for early monitoring of response to ensure a better clinical outcome in schizophrenia patients treated with antipsychotics. It may also provide an early indication for cases in which switching of antipsychotics may be required.

Limitations. The findings need to be validated in analyses of further, independent cohorts, in particular in the light of the regression towards the mean effect observed in this follow-up study. Also, the prediction of positive symptom improvement may be of limited value due to the high proportion of patients who improved after treatment. It will be important to validate these findings using a cohort of responsive and non-response patients. Finally, it is not clear whether any of the changes in biomarker profiles are involved in the mechanism of action of antipsychotics or if they are a consequence of changes that occur in the brain. Studies, which have found that hyperinsulinemia can affect cognition, provide some evidence that the response of some molecules could be mechanism based. However, further studies will be required to answer this question.

Acknowledgements. This research was supported by the Stanley Medical Research Institute (SMRI) and the European Union FP7 SchizDX research programme (Grant reference 223427).

1. Buckley PF, Friedman L, Krowinski AC, Eaton Y, Tronetti M, Miller DD. Clinical and biochemical correlates of "high-dose" clozapine therapy for treatment-refractory schizophrenia. Schizophr Res 2001; 49: 225-227.

2. Chakos M, Lieberman J, Hoffman E, Bradford D, Sheitman B. Effectiveness of secondgeneration antipsychotics in patients with treatment-resistant schizophrenia: a review and meta-analysis of randomized trials. Am J Psychiatry 2001; 158: 518-526.

3. Wong EH, Yocca F, Smith MA, Lee CM. Challenges and opportunities for drug discovery in psychiatric disorders: the drug hunters' perspective. Int J Neuropsychopharmacol 2010; 13: $1269-1284$

4. Mcllwain ME, Harrison J, Wheeler AJ, Russell BR. Pharmacotherapy for treatmentresistant schizophrenia. Neuropsychiatr Dis Treat 2011; 7: 135-149.

5. Samuels BA, Leonardo ED, Gadient R, Williams A, Zhou J, David DJ et al. Modeling treatment-resistant depression. Neuropharmacology 2011; 61: 408-413.

6. Butterfield LH, Palucka AK, Britten CM, Dhodapkar MV, Hakansson L, Janetzki S et al. Recommendations from the iSBTC-SITC/FDA/NCl Workshop on Immunotherapy Biomarkers. Clin Cancer Res 2011; 17: 3064-3076.

7. Hoggatt J. Personalized medicine-trends in molecular diagnostics: exponential growth expected in the next ten years. Mol Diagn Ther 2011; 15: 53-55.

8. Schwarz E, Guest PC, Rahmoune H, Harris LW, Wang L, Leweke FM et al. Identification of a biological signature for schizophrenia in serum. Mol Psychiatry 2011; e-pub ahead of print.

9. Schwarz E, Izmailov R, Spain M, Barnes A, Mapes JP, Guest PC et al. Validation of a blood-based laboratory test to aid in the confirmation of a diagnosis of schizophrenia. Biomark Insights 2010; 5: 39-47.

10. Bossuyt PM, Reitsma JB. The STARD initiative. Lancet 2003; 361: 71

11. Benjamini $Y$, Hochberg $Y$. Controlling the false discovery rate: a practical and powerful approach to multiple testing. J Roy Statist Soc Ser B 1995; 57: 289-300.

12. Barnett AG, van der Pols JC, Dobson AJ. Regression to the mean: what it is and how to deal with it. Int J Epidemiol 2005; 34: 215-220.

13. Breiman L. Random forests. Machine Learning 2001; 45: 5-32.

14. Bland JM, Altman DG. Regression towards the mean. BMJ 1994; 308: 1499.

15. Meyer JM. Effects of atypical antipsychotics on weight and serum lipid levels. J Clin Psychiatry 2001; 62(Suppl 27): 27-34; discussion 40-21. 
16. Yang Y, Wan $\mathrm{C}, \mathrm{Li} \mathrm{H}, \mathrm{Zhu} \mathrm{H}, \mathrm{La} Y, \mathrm{Xi} Z$ et al. Altered levels of acute phase proteins in the plasma of patients with schizophrenia. Anal Chem 2006; 78: 3571-3576.

17. Meltzer HY, Fang VS. The effect of neuroleptics on serum prolactin in schizophrenic patients. Arch Gen Psychiatry 1976; 33: 279-286.

18. Guest PC, Schwarz E, Krishnamurthy D, Harris LW, Leweke FM, Rothermundt M et al. Altered levels of circulating insulin and other neuroendocrine hormones associated with the onset of schizophrenia. Psychoneuroendocrinology 2011; 36: 1092-1096.

19. Meyer JM, Stahl SM. The metabolic syndrome and schizophrenia. Acta Psychiatr Scand 2009; 119: 4-14.

20. Meltzer HY, Lee MA, Jayathilake K. The blunted plasma cortisol response to apomor phine and its relationship to treatment response in patients with schizophrenia. Neuropsychopharmacology 2001; 24: 278-290.

21. Czobor P, Volavka J, Sheitman B, Lindenmayer JP, Citrome L, McEvoy J et al. Antipsychotic-induced weight gain and therapeutic response: a differential association. J Clin Psychopharmacol 2002; 22: 244-251.

22. Meltzer HY, Perry E, Jayathilake K. Clozapine-induced weight gain predicts improvement in psychopathology. Schizophr Res 2003; 59: 19-27.

23. Girard J. Is leptin the link between obesity and insulin resistance? Diabetes Metab 1997; 23(Suppl 3): 16-24.

24. Kajikawa Y, Ikeda M, Takemoto S, Tomoda J, Ohmaru N, Kusachi S. Association of circulating levels of leptin and adiponectin with metabolic syndrome and coronary heart disease in patients with various coronary risk factors. Int Heart J 2010; 52: 17-22.

25. Kim BJ, Sohn JW, Park CS, Hahn GH, Koo J, Noh YD et al. Body weight and plasma levels of ghrelin and leptin during treatment with olanzapine. J Korean Med Sci 2008; 23: 685-690.
26. Zhang ZJ, Yao ZJ, Liu W, Fang Q, Reynolds GP. Effects of antipsychotics on fat deposition and changes in leptin and insulin levels. Magnetic resonance imaging study of previously untreated people with schizophrenia. Br J Psychiatry 2004; 184: 58-62.

27. Sato $T$, Hanyu H, Hirao K, Kanetaka H, Sakurai H, Iwamoto T. Efficacy of PPAR-gamma agonist pioglitazone in mild Alzheimer disease. Neurobiol Aging 2011; 32: 1626-1633.

28. Pannacciulli N, Le DS, Chen K, Reiman EM, Krakoff J. Relationships between plasma leptin concentrations and human brain structure: a voxel-based morphometric study. Neurosci Lett 2007; 412: 248-253.

29. Guest PC, Wang L, Harris LW, Burling K, Levin Y, Ernst A et al. Increased levels of circulating insulin-related peptides in first-onset, antipsychotic naive schizophrenia patients. Mol Psychiatry 2010; 15: 118-119.

30. Arranz B, Rosel P, Ramirez N, Duenas R, Fernandez P, Sanchez JM et al. Insulin resistance and increased leptin concentrations in noncompliant schizophrenia patients but not in antipsychotic-naive first-episode schizophrenia patients. J Clin Psychiatry 2004; 65 : 1335-1342.

Translational Psychiatry is an open-access journal published by Nature Publishing Group. This work is licensed under the Creative Commons Attribution-Noncommercial-No Derivative Works 3.0 Unported License. To view a copy of this license, visit http://creativecommons.org/licenses/by-nc-nd/3.0/

\section{Supplementary Information accompanies the paper on the Translational Psychiatry website (http://www.nature.com/tp)}

